\title{
Anisotropic magnetoresistive study in bilayer NiFe-NiO for sensor applications
}

\author{
Carlos Morón , Alfonso Garcia ，Enrique Tremps , \\ Jose Andrés Somolinos
}

Keywords: anisotropic, magnetoresistance, bilayer.

\begin{abstract}
Related with the detection of weak magnetic fields, the anisotropic magnetoresistive (AMR) effect is widely utilized in sensor applications. Exchange coupling between an antiferromagnet (AF) and the ferromagnet (FM) has been known as a significant parameter in the field sensitivity of magnetoresistance because of pinning effects on magnetic domain in FM layer by the bias field in AF. In this work we have studied the thermal evolution of the magnetization reversal processes in nanocrystalline exchange biased $\mathrm{Ni}_{80} \mathrm{Fe}_{20} / \mathrm{Ni}-\mathrm{O}$ bilayers with large training effects and we report the anisotropic magnetoresistance ratio arising from field orientation in the bilayer.
\end{abstract}

\section{Introduction}

Related with the detection of weak magnetic fields, the anisotropic magnetoresistive (AMR) effect is widely utilized in sensor applications. Various sensor designs and electronic evaluation circuits have been developed to overcome temperature dependence, offset, and hysteresis $[1,2]$.

Exchange coupling between an antiferromagnet (AF) and the ferromagnet (FM) has been known as a significant parameter in the field sensitivity of magnetoresistance because of pinning effects on magnetic domain in FM layer by the bias field in AF [3].

The AF $\mathrm{NiO}$ film has received some attention in giant and anisotropic magnetoresistance (GMR, AMR) because of their structural stability [4]. Much effort has been made on the exchange biasing field in $\mathrm{NiFe} / \mathrm{NiO}$ system, relating to the influencing parameters involved during fabrication process such as structure, annealing and surface roughness by measuring $M-H$ loop and MR profile under the magnetization in easy- and hard-axis direction.

In this work we have studied the thermal evolution of the magnetization reversal processes in nanocrystalline exchange biased $\mathrm{Ni}_{80} \mathrm{Fe}_{20} / \mathrm{Ni}-\mathrm{O}$ bilayers with large training effects and we report the anisotropic magnetoresistance ratio arising from field orientation in the bilayer [5].

\section{Experimental and results}

$\mathrm{Ni}_{80} \mathrm{Fe}_{20}(20 \mathrm{~nm}) / \mathrm{Ni}-\mathrm{O}(20 \mathrm{~nm})$ bilayers were deposited by RF sputtering technique onto glass substrates kept at room temperature in $\mathrm{Ar}$ atmosphere. TEM structural characterization revealed typical grain sizes of around $4 \mathrm{~nm}$ for $\mathrm{Ni}-\mathrm{O}$ layers and $14 \mathrm{~nm}$ for $\mathrm{Ni}_{80} \mathrm{Fe}_{20}$ grains.

Figure 1 shows hysteresis loop measured at $400 \mathrm{~K}$; both exhibiting the typical displacement towards negative fields. This hysteresis loop does not present displacement and the value of the coercive field is $7 \mathrm{Oe}$. This shows that there is exchange coupling at this temperature between the oxide and Permalloy.

After heating the sample at $450 \mathrm{~K}$ we proceeded to cooling with a magnetic field applied of 900 Oe. The cycle Hysteresis measured at $50 \mathrm{~K}$ has a value of the coercivity field, Hc, 63 Oe and a value of the displacement field, $\mathrm{H}_{\mathrm{e}},-48 \mathrm{Oe}$, as shown in Figure 2. 


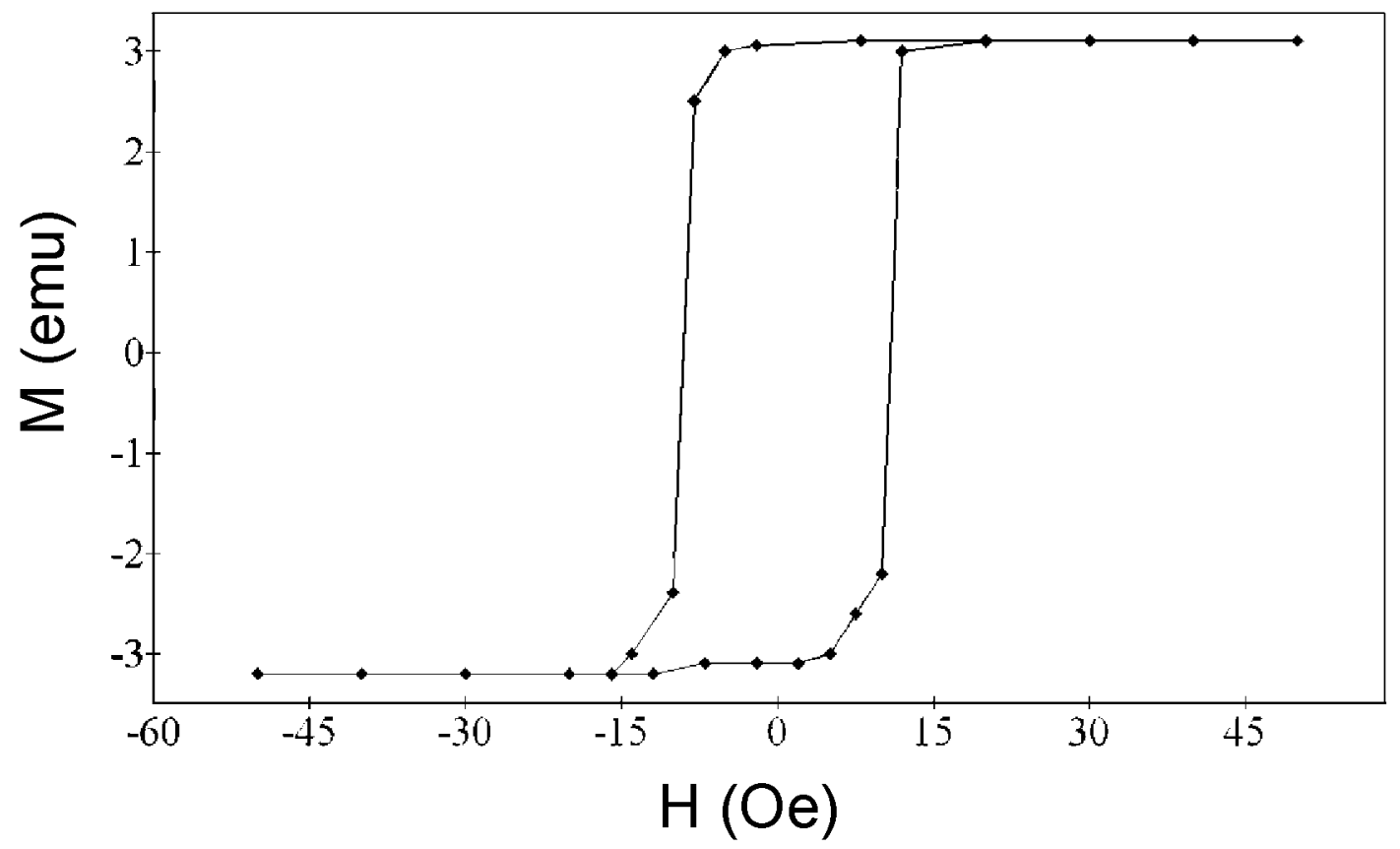

Fig. 1. Hysteresis loop measured at a temperature of $400 \mathrm{~K}$.

Hysteresis loops were measured at different temperatures, occurring two temperature ranges in which the behaviour becomes different $\mathrm{H}_{\mathrm{e}}$ field. Between $50 \mathrm{~K}$ and $300 \mathrm{~K}$ the displacement that presented is the usual, ie, negative and its value decreases progressively with temperature increase. The coercive field also decreases with temperature. However, above $300 \mathrm{~K}$ there is a change of sign in displacement of the hysteresis loop, which is positive throughout the range. The value $\mathrm{H}_{\mathrm{e}}$ maximum is reached at $350 \mathrm{~K}$, temperature from which $\mathrm{H}_{\mathrm{e}}$ starts decrease until it becomes zero at $400 \mathrm{~K}$. All these results can be seen by the values of $\mathrm{H}_{\mathrm{E}}$ and $\mathrm{H}_{\mathrm{c}}$ shown in Figure 3 and Figure 4 .

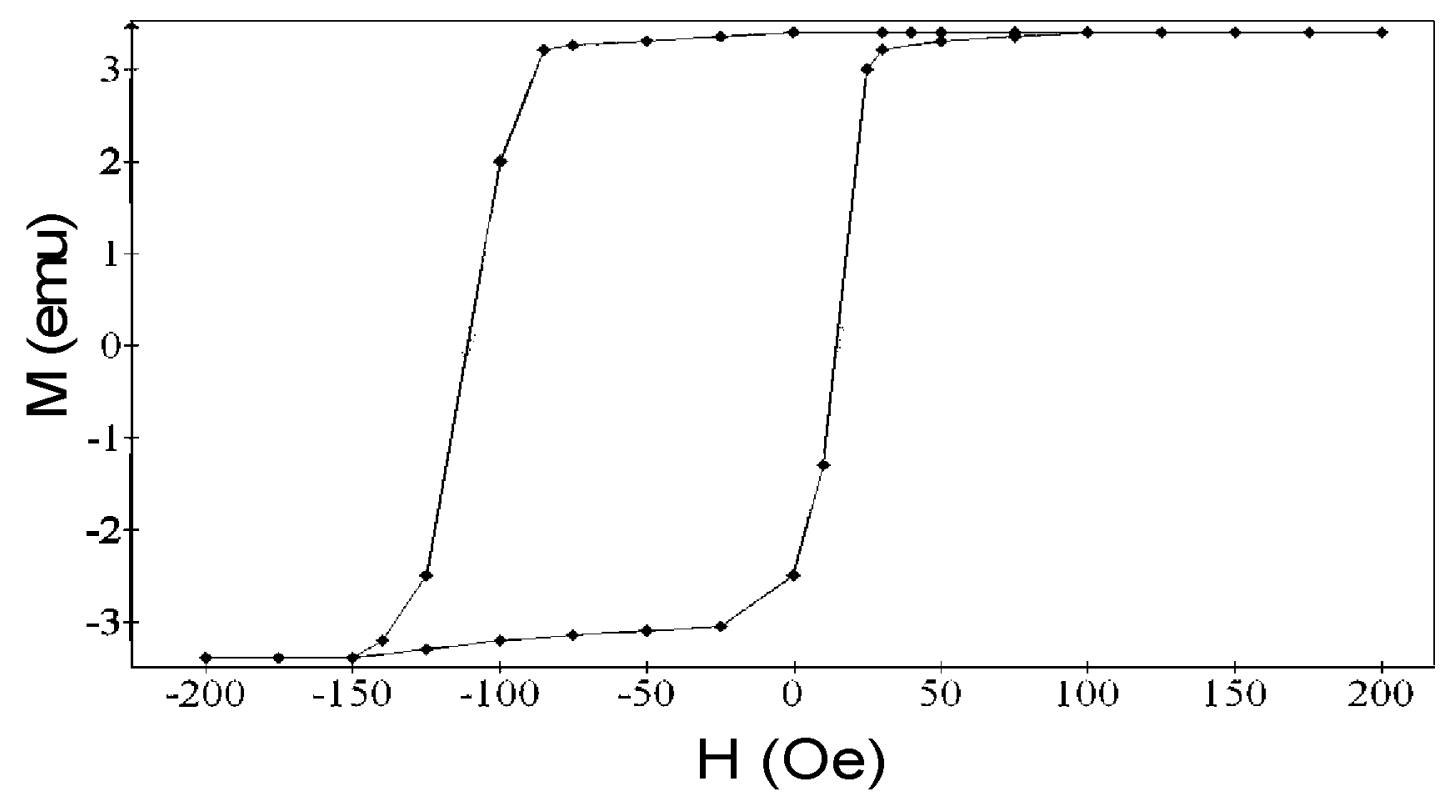

Fig. 2. Hysteresis loop measured at a temperature of $50 \mathrm{~K}$. 


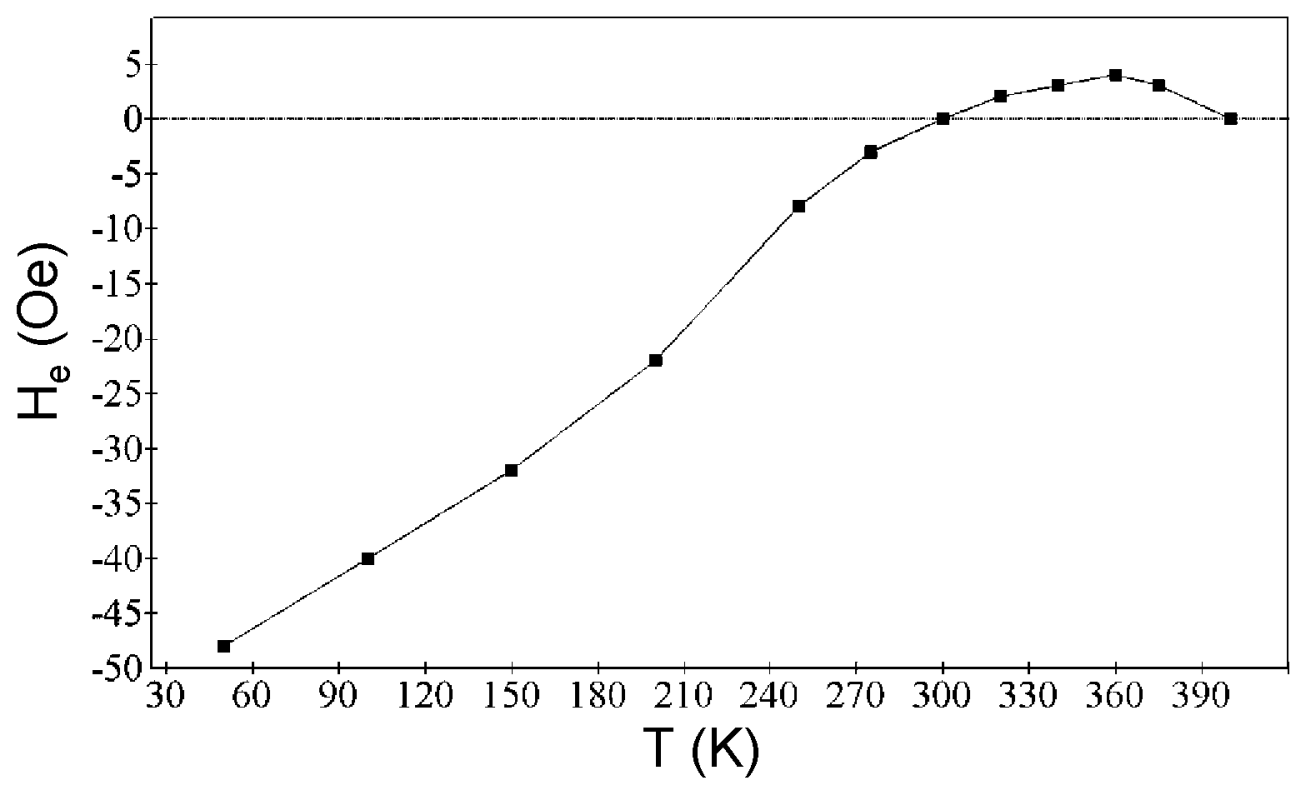

Fig.3. Thermal evolution of the exchange field of the sample after cooling with a magnetic field applied of $900 \mathrm{Oe}$.

The magnetoresistance (MR) ratio, $\Delta R / R_{\mathrm{s}}$ was measured by the four-probe method as a function of magnetizing angle, $\theta$ from the bias field, where the MR was measured at perpendicular direction to the bias field. The external magnetic field of 90 Oe was applied by a planar solenoid, of which positive direction was the same as that of the bias field.

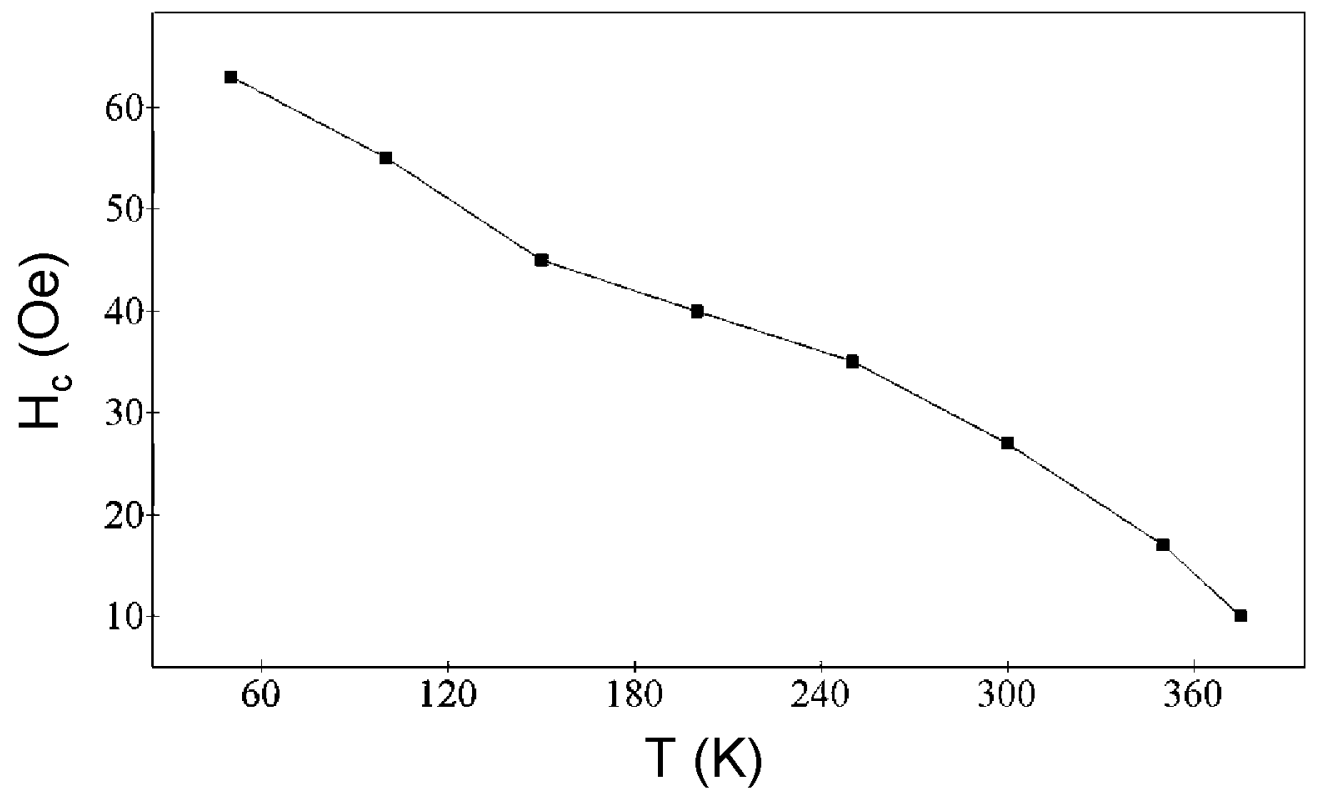

Fig.4. Thermal evolution of the coercitive field of the sample after cooling with a magnetic field applied of 900 Oe.

The measurement of the AMR was made on the sample polarized initial esay axis, which is also the current direction. Magnetoresistance curves were measured with a maximum applied field of \pm 90 Oe depending on the angle with the easy axis. Then we have obtained that the maximum of this variation occurs in the direction $90^{\circ}-270^{\circ}$ were that value is around $1 \%$. The minimum value, approximately $0.3 \%$, occurs in the easy axis. 


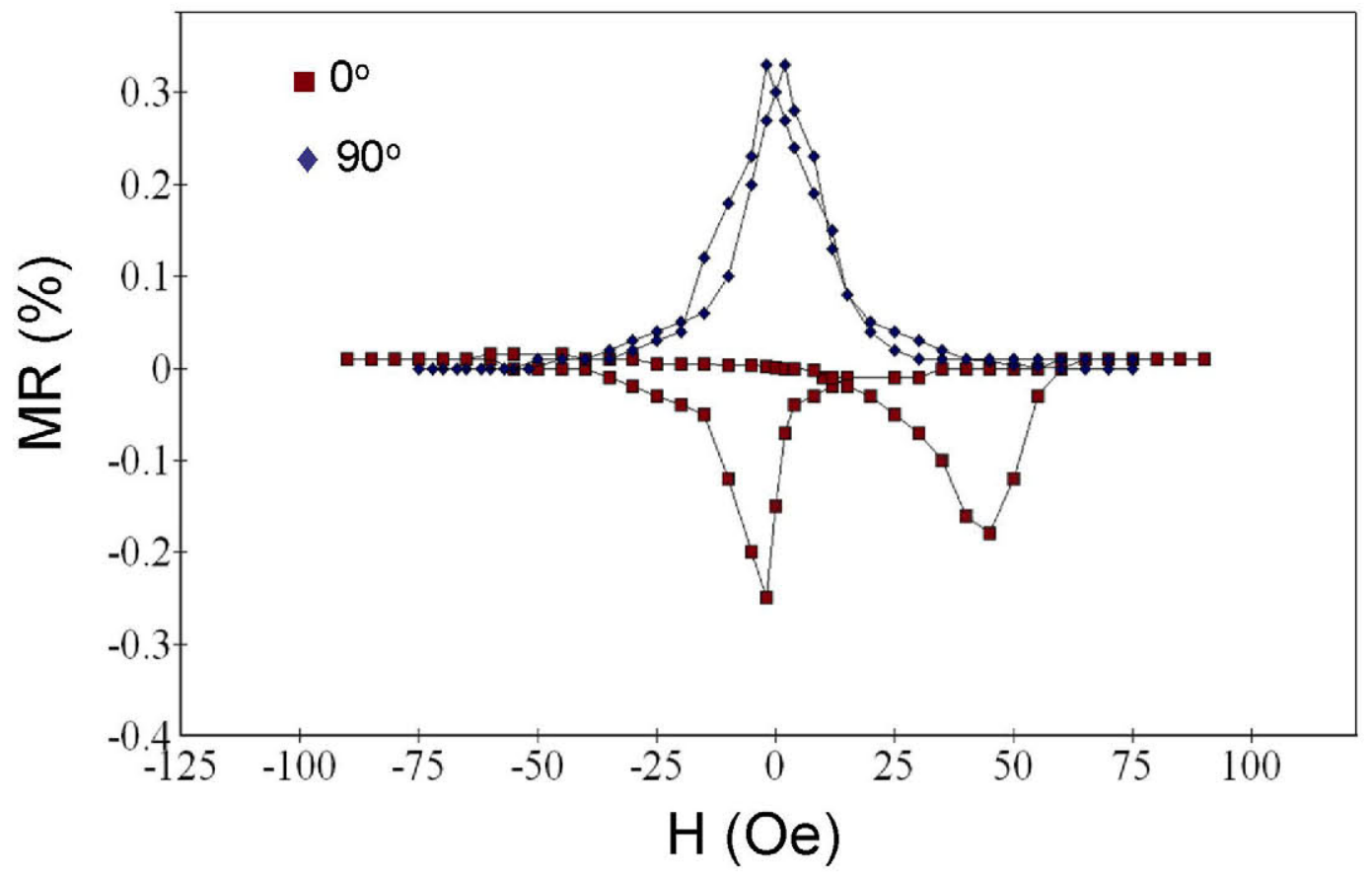

Fig.5. Marnetoresistance measured with and without exchange field.

\section{Summary and conclusions}

In summary, $\mathrm{Ni}_{80} \mathrm{Fe}_{20}(20 \mathrm{~nm}) / \mathrm{Ni}-\mathrm{O}(20 \mathrm{~nm})$ bilayers were deposited by RF sputtering technique onto glass substrates kept at room temperature in $\mathrm{Ar}$ atmosphere. TEM structural characterization revealed typical grain sizes of around $4 \mathrm{~nm}$ for $\mathrm{Ni}-\mathrm{O}$ layers and $14 \mathrm{~nm}$ for $\mathrm{Ni}_{80} \mathrm{Fe}_{20}$ grains. The magnetoresistance (MR) ratio, $\Delta R / R_{\mathrm{S}}$ was measured by the four-probe method as a function of magnetizing angle, $\theta$ from the bias field, where the MR was measured at perpendicular direction to the bias field. The external magnetic field of 90 Oe was applied by a planar solenoid, of which positive direction was the same as that of the bias field. Then we have obtained that the maximum of the magnetoresistance variation occurs in the direction $90^{\circ}-270^{\circ}$ were that value is around $1 \%$. The minimum value, approximately $0.3 \%$, occurs in the easy axis.

\section{References}

[1] U. Dibbern, Magnetoresistive sensors, in: W. GoK pel, J. Hesse, J. N. Zemel (Eds.), Sensors, Volume 5: Magnetic Sensors (R. Boll, K. J. Overshott (Vol. Eds.)), VCH Verlagsgesellschaft, Weinheim, pp. 341-380 (1989).

[2] D.J. Mapps, Y.Q. Ma, M.A. Akhter, Sensors Actuators A 81, 60 (2000).

[3] S.S. Lee, D.G. Hwang, C.M. Park, K.A. Lee, J.R. Lee, J. Appl. Phys. 81, 5298 (1997).

[4] P. Ripka, M. Vopalenski, A. Platil, M. Döscher, K.-M.H. Lenssen, H. Hauser, J. Magn. Mag. Mat. 254-255, 639 (2003).

[5] E. Pina, C. Prados, A. Hernando. J. Mag. Mag. Mat. 272-276, 1905 (2004). 
Materials and Applications for Sensors and Transducers II

10.4028/www.scientific.net/KEM.543

Anisotropic Magnetoresistive Study in Bilayer NiFe-NiO for Sensor Applications

10.4028/www.scientific.net/KEM.543.167 